\title{
Major Depression During and After the Menopausal Transition: Study of Women's Health Across the Nation (SWAN)
}

\author{
Joyce T. Bromberger, PhD, \\ University of Pittsburgh, Pittsburgh, Pennsylvania, Departments of Epidemiology and Psychiatry \\ Howard M. Kravitz, DO, MPH, \\ Rush University Medical Center, Chicago, Illinois, Department of Psychiatry and Department of \\ Preventive Medicine \\ Yue-Fang Chang, PhD, \\ University of Pittsburgh, Pittsburgh, Pennsylvania, Department of Neurosurgery \\ Jill M. Cyranowski, PhD, \\ University of Pittsburgh, Pittsburgh, Pennsylvania, Department of Psychiatry \\ Charlotte Brown, PhD, and \\ University of Pittsburgh, Pittsburgh, Pennsylvania, Department of Psychiatry \\ Karen A. Matthews, PhD \\ University of Pittsburgh, Pittsburgh, Pennsylvania, Department of Psychiatry, Epidemiology, and \\ Psychology
}

\section{Abstract}

Background-It is unclear whether risk for major depression during the menopausal transition or immediately thereafter is increased relative to premenopause.

Objectives-To examine whether the odds of experiencing major depression were greater when women were perimenopausal or postmenopausal compared to when they were premenopausal, independent of a history of major depression at study entry and annual measures of vasomotor symptoms, serum levels or changes in estradiol, follicular stimulating hormone, or testosterone and relevant confounders.

Methods-Participants included the 221 African American and Caucasian women, aged 42-52, who were premenopausal at entry into the Pittsburgh site of a community-based study of menopause, the Study of Women's Health Across the Nation (SWAN). We conducted the Structured Clinical Interview for DSM-IV Axis I Disorders (SCID) to assess diagnoses of lifetime, annual, and current major depression at baseline and annual follow-ups. Psychosocial and health factors, and blood samples for assay of reproductive hormones were obtained annually.

Results-Women were two to four times more likely to experience major depression episode when they were perimenopausal or early postmenopausal. Repeated measures logistic regression analyses showed that the effect of menopausal status was independent of history of major depression and annually measured upsetting life events, psychotropic medication use, vasomotor symptoms and serum levels of or changes in reproductive hormones. History of major depression was a strong predictor of major depression throughout the study.

Corresponding Author and Reprints: Dr. Joyce T. Bromberger, Ph.D., University of Pittsburgh, 3811 O'Hara Street, Pittsburgh, PA 15213; tel. 412-648-7108; fax 412-383-5123; brombergerjt@upmc.edu.

All authors report no conflict of interest. 
Conclusions-The risk of major depression is greater for women during and immediately after the menopausal transition than when they are premenopausal.

\section{Introduction}

In the first half of the $20^{\text {th }}$ century, the common wisdom based on clinical and anecdotal reports was that menopause conferred an increased risk of depression. Indeed, the Diagnostic and Statistical Manual of Mental Disorders, Second Edition, included a mood disorder called Involutional Melancholia, a diagnosis specific to menopause, that was subsequently removed from the Diagnostic and Statistical Manual of Mental Disorders, Third Edition, because of the lack of data to support its existence (Weissman, 1979). In the latter two decades of the century, numerous, mostly cross-sectional studies, reported inconsistent findings with the majority reporting either no association or an association between the menopausal transition and depressive symptoms (Matthews et al, 1990; Hunter, 1992; Kaufert et al, 1992; Avis et al, 1994; O'Connor et al, 1994; Kuh et al, 1997; Maarten et al, 2002; Freeman et al, 2004). Specifically, we have moved from the "belief" that women were particularly susceptible to depression after the menopausal transition to the current empirically supported conclusion that middle-aged women are at a greater risk for depression during the transition than before (Maarten et al, 2002; Freeman et al, 2004; Cohen et al, 2006; Bromberger et al, 2007). Three limitations in the literature to date are important. First, the conclusions are based primarily on longitudinal studies of depressive symptoms, not depression diagnoses, albeit using standard instruments, such as the Center for Epidemiological Studies of Depression Scale (CES-D). Second, the longitudinal data on the risk for depression immediately after the transition (postmenopause) are inconclusive because few studies follow women beyond the final menses for any length of time. Third, it is unclear whether any obtained effects are due to a lifetime history of elevated risk for depression as most studies do not have data with regard to past history.

Also unclear is whether the reproductive hormones that change markedly during the menopausal transition, i.e. gonadotropins and ovarian hormones, are associated with major depression. Increased odds of high depressive symptoms were associated with variability in serum estradiol (E2) and follicle-stimulating hormone (FSH) levels in one study (Freeman et $\mathrm{al}, 2006)$ and with declines in serum E2 and large increases in FSH levels in another (Schmidt et al, 2002). Neither study found a significant association between odds of depressive symptoms and absolute hormone levels.

The Study of Women's Health Across the Nation (SWAN) Pittsburgh site provides an opportunity to address three questions: (1) Does the risk for a major depressive episode (MDE) increase during the menopausal transition or immediately thereafter and is the risk independent of a history of major depression at study entry and upsetting events at annual visits? (2) Do annually measured reports of 'frequent' vasomotor symptoms or circulating levels of or changes in reproductive hormones, E2, FSH, testosterone (T)) account for or attenuate the association of menopausal status and major depression? and (3) If the risk for an episode during the postmenopause increases, how long is the period of increased risk? We also explored whether risk for a MDE varied by race (African Americans or Caucasians).

\section{Method}

\section{Subjects and Procedures}

This study was conducted among participants in the Pittsburgh site of SWAN, a multisite community based cohort investigation of the menopause and aging. Of the 463 women enrolled in Pittsburgh SWAN, 443 participated in the SWAN ancillary study, the Mental 
Health Study (MHS). At study entry, about half the women were premenopausal and half were early perimenopausal. The sample for the current analyses consisted of the 77 African American and 101 Caucasian women, aged 42-52 years, who were premenopausal at study entry. We selected this subset to provide a more conservative estimate of the relative odds of experiencing a major depressive episode during and after the menopausal transition compared to premenopause. In the Mental Health Study, psychiatric interviews were conducted to obtain information on lifetime and current psychiatric disorders in addition to the extensive data collected as part of the larger SWAN study, which tracks a variety of health parameters in a multiethnic community based sample of women as they transition through menopause.

The sampling procedures and design of SWAN have been described previously (Sowers et al, 2000). Each site recruited Caucasian women and a sample of a predetermined minority group. Pittsburgh participants were recruited using random digit dialing which was supplemented by a voter's registration list and enrolled 162 African American women and 301 Caucasian women. Eligibility criteria for SWAN included being aged between 42 and 52 , having an intact uterus, having had at least one menstrual period and no use of reproductive hormones in the previous 3 months, and self-identifying with one of the site's designated race/ethnic groups. In Pittsburgh, 3,540 telephone numbers called were of unknown usability (e.g., busy signals, never home, moved etc), 2,148 contacts yielded unknown cohort eligibility (e.g., incomplete screening interview, refusal to be screened), 12,027 women were ineligible to be screened (e.g., out of age range, no period in past 3 months), and 2,604 completed the screening interview. Of the latter, 1,050 were eligible and 463 of those eligible entered the SWAN study in Pittsburgh. Participants and those who were eligible but did not participate did not vary by ethnicity, marital status, parity, quality of life, social support, perceived stress or reports of feeling "blue or depressed" in the prior two weeks measured during the screener. There were no significant differences between the 443 Mental Health Study participants and the 20 non-participants with respect to sociodemographic factors and Center for Epidemiological Studies of Depression Scale (CES-D) scores of 16 or higher.

At the beginning of the study all participants signed an informed consent in accordance with the University of Pittsburgh Institutional Review Board guidelines. The women provided extensive health, psychosocial, lifestyle and biologic data at baseline and at annual followup visits as part of the larger SWAN study. Height and weight were measured using a common protocol and body mass index (BMI) was calculated $\left(\mathrm{kg} / \mathrm{m}^{2}\right)$. Women were scheduled for venipuncture for assays of reproductive hormones prior to 10 am on days 2-5 of a spontaneous menstrual cycle occurring within 60 days of recruitment at the baseline visit, and annually thereafter. If a timed sample could not be obtained after two attempts, a random fasting sample was taken within a 90-day window of the anniversary of the baseline visit. The Structured Clinical Interview for the Diagnosis of DSM-IV Axis I Disorders (SCID) was administered 2 to 9 months after the SWAN baseline assessment and annually within three months of the annual SWAN visit.

\section{Measures}

Assessment of Psychiatric Disorders-Diagnoses of lifetime and current major depressive disorders were determined from interviews conducted by trained clinicians utilizing the SCID (Spitzer et al, 1992). The SCID has been used with many different ethnic groups and extensive field-testing has demonstrated its suitability for research purposes; adequate reliability has been demonstrated in numerous studies (Williams et al, 1992). All interviewers had extensive clinical experience and at least a master's degree in a relevant field, e.g., social work, psychology. Interviewers were supervised by the first author (JTB). 
Extensive training and qualitative procedures were used to ensure and monitor consistency of SCID administration, symptom elicitation, and diagnostic decision-making. These included central training conducted by Biometrics, follow up practice with community volunteers, and ongoing supervision by the principal investigator (JTB). All interviews with study participants were audiotaped. Tapes were used for supervision, to monitor rater drift and to estimate interrater reliability. Using a systematic sampling procedure, 24-36 audiotapes were selected and reviewed by all interviewers and supervisor (JTB) and consultant (HMK) to estimate interrater reliability for lifetime diagnoses of selected disorders and subsequent follow-up diagnoses. Interrater reliability was very good to excellent for lifetime major depressive disorder $($ Kappa $=.81)$ and for major depression in the past year (kappa $=.76-.89$, reliability at several follow-ups).

A history of major depression was defined as the occurrence of major depression prior to the SWAN baseline assessment. Annual SCIDs assessed major depressive episodes that occurred in the previous year or were current at the baseline or annual assessment.

Participants with current major depression were asked by the interviewer whether they were receiving or had considered seeking help for the distress/depression they were experiencing. If treatment had not been obtained, information regarding resources available in the community was offered. The presence of current suicidal ideation is assessed as part of the Mood Disorders module of the SCID. The SCID Manual of Operations includes procedures to intervene and arrange for emergency referrals if women reported suicidal thoughts during the interview.

\section{Independent variables}

Menopausal status-Menopausal status was based on menstrual bleeding patterns in the previous 12 months and was categorized as the following: a) premenopausal - menstrual period in the past 3 months with no change in regularity in the past 12 months); b) menopausal transition/perimenopausal - menstrual period in the past 3 months with change in regularity over the previous 12 months or no menstrual period within the past 3 months, but some menstrual bleeding within the past 12 months; c) post menopausal - no menstrual period within the past 12 months. The classifications are similar to those recommended by the World Health Organization (World Health Organization, 1996). Based on SWAN eligibility requirements, all women were premenopausal or early perimenopausal at baseline. As noted, the women in the current analyses were all premenopausal at baseline.

Vasomotor symptoms-Data on vasomotor symptoms (VMS: hot flashes or night sweats) were obtained with a self-report checklist of symptoms commonly included in studies of the menopause (Neugarten et al, 1965; McKinlay et al, 1974; Matthews et al, 1994). At each visit, women were asked how often they had experieinced hot flashes and night sweats in the past 2 weeks: not at all, $1-5$ days, 6-8 days, 9-13 days, daily. We defined frequent VMS as those occurring at least 6 days in the past 2 weeks.

Reproductive hormones-The fasting blood draw was targeted to days 2 to 5 of the follicular phase of the menstrual cycle in menstruating women and within 90 days of the anniversary of the baseline examination date. If a timed sample could not be obtained after two attempts, a random fasting sample was taken within a 90-day window of the annual visit. Blood was refrigerated within 1 to 2 hours after phlebotomy. All samples were maintained at $4{ }^{\circ} \mathrm{C}$ until separated; following centrifugation, the serum was aliquotted, frozen at $-80^{\circ} \mathrm{C}$, and shipped on dry ice to the central laboratory, the SWAN Endocrine Laboratory, at the University of Michigan, Ann Arbor, using the ACS-180 automated analyzer (Bayer Diagnostics Corp where all assays were performed. All assays used a 
chemiluminescent immunoassay with a solid-phase anti-(Ig)G immunoglobulin conjugated to paramagnetic particles, anti-ligand antibody, and competitive ligand labeled with dimethylacridinium ester. E2 assays were conducted in duplicate and FSH and T in singlicate. The respective intra-assay and inter-assay coefficients of variation were $8.5 \%$ and $13.8 \%$ for E2, $12.0 \%$ and $6.0 \%$ for FSH and $9.7 \%$ and $11.3 \%$ for T,) (Randolph et al, 2005).

Covariates-Demographic factors relevant to the current analyses included baseline marital status, level of educational attainment and age at annual visit. Marital status was categorized as single, married/partnered, or separated/divorced/widowed. Educational attainment was classified in three categories, completed high school or less, high school plus some college, or completed college or more. Race/ethnicity was self-identified based on the participant's response to the question "what is your primary racial or ethnic group?" At each visit, women completed a checklist of 18 negative life events indicating if any of these had occurred in the past year and, if so, to rate these according to how upsetting they were: not at all, somewhat, or very upsetting. Women were categorized as having experienced at least one "very upsetting" event versus none/somewhat upsetting event since their last study visit. Psychotropic medication use in the past year and body mass index at each visit was also considered for inclusion to account for potential confounding with depression.

\section{Statistical Analysis}

The analytic sample consisted of 221 premenopausal women for whom we had 1,698 person years of observations, $89 \%$ of a possible 1,913 , over the ten years of assessments. The possible number of observations was based on the number of women expected at each visit and whose data were eligible for inclusion. For example, premenopausal or perimenopausal women who were using HT at a visit were not considered as possible observations because their data from that visit were excluded as noted below. On the other hand, a missed visit would have been included as a possible person year of observation.

We used repeated measures random effects logistic regression models to examine whether the menopausal transition was associated with an increased odds of meeting criteria for a MDE in the past year or currently at each annual visit. The inclusion of a (woman-specific) random intercept allows for the correlation between all observations from a given woman to be modeled and enables us to ascribe a 'woman-specific' interpretation to model parameters. Random effects logistic regression models are relatively robust to missing data and make use of all available data across all visits. Data from women who reported a surgical menopause were censored at that visit forward and data from those who were premenopausal or perimenopausal and were using hormone therapy (HT) were excluded at the visit at which they reported its use because HT use affects bleeding patterns and obfuscates menopausal status. The data from these HT users were included at subsequent visits when they had not been using HT for one year and menopausal status could be determined. Data from postmenopausal women using HT were included.

We first used random effects logistic regression analyses to examine the odds of a MDE for each of the following variables individually: marital status and education, and the time dependent variables: upsetting life events, psychotropic medication use, BMI, hormone therapy use (during postmenopause), VMS, and levels of natural log transformed hormones and change in log hormones from baseline. Variables associated with MDE at $\mathrm{p}<.10$ in these univariate analyses were candidates for inclusion in the multivariable models. To examine our first aim, we modeled the odds of having a MDE at any given annual assessment or in the previous year as a function of the participant's menopausal status, age, race, and history of major depression at study entry. Race was included as an independent variable because of the study design. We next added the covariates associated with MDE at $p<.10$ in the 
univariate analyses to control for their confounding with depression. To address our second aim, we added to the model individually, frequent VMS and endogenous hormones that were significant at a p-value $<.10$ in univariate analyses. For our third aim, we divided postmenopause into early ( $\leq 2$ years amenorrheic) and late ( $>2$ years amenorrheic) postmenopause and conducted analyses as described above. Analyses were run using SAS version 8 statistical software (SAS Institute, Inc., Cary, North Carolina) and Stata version 7statistical software (StataCorp LP, College Station, Texas).

\section{Results}

All women had at least one perimenopause visit and 131 had at least one post visit with the numbers of visits for each participant during each stage of menopause ranging from 1 to 10 . Among those who reached postmenopause during the study, the average number of visits was 8.98 out of 10 possible visits.

Figure 1 shows the total number of actual person-years of observations and the numbers of observations during each menopausal stage for the 221 participants. The total number of major depressive episodes (MDEs) and the number diagnosed during each stage are also shown. It can be seen that there were a total of 146 MDEs diagnosed during 1,698 annual visits; 31 occurred during a premenopause visit, 71 during a perimenopause visit, and 44 during a postmenopause visit. Thus, MDEs were diagnosed during $5.8 \%(\mathrm{n}=31)$ of 535 premenopause visits, $9.1 \%(\mathrm{n}=71)$ of 782 perimenopause visits, and $9.8 \%(\mathrm{n}=44)$ of 381 postmenopause visits. Over the 10 years of the study, $69(23.8 \%)$ women had at least one MDE: $33.8 \%$ of African Americans and 29.9\% of Caucasians. Of these, $15(21.7 \%)$, had 3 or more episodes, $6(14.0 \%)$ Caucasian women and $9(34.6 \%)$ African American women.

At baseline women with and without at least one MDE over the study did not differ by race, marital status or education. Nor did they vary by prevalence of frequent VMS, log E2 or log FSH. Women who ever had an MDE had a significantly higher proportion with very upsetting life events in the past year, current use of psychotropic medications, a history of major depression and a lower mean log testosterone than those who never had an MDE.

(Table 1) Because reproductive hormone levels have been shown to vary by BMI (Burger et al, 1999), analyses for each hormone was also adjusted for BMI. This did not change substantively the association of hormones with MDE.

In the univariate repeated measures analyses (Table 2), being perimenopausal or postmenopausal, compared to being premenopausal, was associated with a significantly greater odds of experiencing an MDE over time. Also significant were age, history of major depression, use of psychotropic medications, very upsetting life events, BMI and frequent vasomotor symptoms. One reproductive hormone, log FSH, showed only a non-significant trend $(\mathrm{p}=.08)$; hormone therapy use and race were not significant.

Table 3 displays the results of the three multivariable models. Model A shows that being perimenopausal or postmenopausal compared to being premenopausal was significantly associated with an increased odds of experiencing a MDE and that the ORs increased as women progressed through the transition to postmenopause, independent of age, race, and history of major depression. Model B shows that perimenopause and postmenopause remained significantly related to the development of a new MDE independent of covariates. Having a history of major depression at baseline, psychotropic medication use, higher BMI, and very upsetting life events annually were each associated with odds of a MDE. Model C shows that frequent VMS were marginally significant as $95 \%$ confidence intervals included 1.0 and reduced the odds ratios for peri- and postmenopause compared with premenopause by $4.8 \%$ and $10.6 \%$, respectively. However, peri- and postmenopause remained significant 
predictors of MDEs (ORs= 1.98 and 3.86, respectively). Log FSH was not significant (data not shown).

When the postmenopause period was divided into early ( $\leq 2$ yrs amenorrheic) and late ( $>2$ yrs amenorrheic), the multivariable analyses showed that the early postmenopause posed a significantly greater risk for MDEs than did premenopause $(\mathrm{OR}=5.01,95 \% \mathrm{CI}=1.66,15.17)$ whereas late postmenopause $\operatorname{did}$ not $(\mathrm{OR}=2.93,95 \% \mathrm{CI}=.92,9.36)$. Frequent VMS was marginally significant $(\mathrm{OR}=1.76,95 \% \mathrm{CI}=.99,3.13)$ as was perimenopause $(\mathrm{OR}=1.91$, $95 \% \mathrm{CI}=.99,3.72)$

\section{Discussion}

In longitudinal analyses that spanned 10 years, we found that the odds of experiencing a major depressive episode was significantly greater when women were perimenopausal and postmenopausal than when they were premenopausal, independent of a baseline history of major depression and annually measured very upsetting life events, use of psychotropic medications, reproductive hormone characteristics, BMI, and frequent VMS. These findings are consistent with previous results in the entire SWAN cohort $(\mathrm{n}=3296)$ showing that the odds of high depressive symptoms (CES-D score of 16 or higher) were significantly greater as women progressed through the menopausal transition (Bromberger et al, 2007).

In the last 3 decades, epidemiological studies of menopause have found no relationship between depressive symptoms and menopausal status (Matthews et al, 1994; McKinlay et al, 1994; Kaufert et al, 1992) or in some cases, higher levels of symptoms during perimenopause (Hunter et al, 1992; Kuh et al, 1997; Avis et al, 1994). These studies had numerous methodological limitations, including select or small samples, non-standard measures of depressive symptoms, and inconsistent definitions of menopausal status. Most were cross-sectional. The results of recent longitudinal studies (Freeman et al, 2004; Maarten et al, 2002; Cohen et al, 2006) have more consistently found that the odds of high depressive symptoms were greater during perimenopause than premenopause. Compared to earlier studies, recent studies had larger or more diverse samples, longer and more frequent follow-up, more consistent, careful definitions of menopausal status and used sophisticated analyses that utilized fully the longitudinal data. With one exception (Maarten et al, 2002), these studies did not find an increased risk for depressive symptoms postmenopause. The null findings may have been due to insufficient power because of the relatively small numbers of women followed through postmenopause. For example, the Penn Ovarian Aging study (POA) (Freeman et al, 2004) reported an increase in depressive symptoms in the perimenopause and a subsequent decline postmenopause over 4 years of follow-up. However, in this study $73 \%$ of the 332 participants remained premenopausal over the 4 years of follow-up.

Three longitudinal studies of menopause have assessed depressive disorders (Freeman et al, 2004; Cohen et al, 2006; Schmidt et al, 2004). The POA found that depressive disorders, were not more likely to occur during the menopausal transition than premenopausal (Freeman et al, 2004). However, this study was limited by having only 4 years of data collection, only $3 \%$ of the sample had reached postmenopause, and the assessment of MDE covered only the past month, making the numbers for postmenopause and MDE too small to determine statistical significance. The POA and Harvard Study of Moods and Cycles reported an increased risk of first onset depressive disorder during perimenopause compared to premenopause over 8 years. (Cohen et al, 2006). Importantly, neither study included women who had ceased menstruating and only examined a subset of women. 
Our findings of an increased risk of MDE during postmenopause and particularly during the first two years after the final menstrual period are similar to those reported in a small longitudinal study (5 years) that followed 29 regularly cycling women in their 40s through one year after their last menses (Schmidt et al, 2004). In this study, every 3-6 six months detailed measures of menstrual cycles, symptoms, and depression as well as plasma FSH were collected and the SCID was conducted to obtain data on episodes of minor and major depression. Results indicated that women were significantly more likely to experience an episode of clinical depression (primarily minor depression) during the year before or after their final menstrual period than when they were premenopausal.

In our sample, the increased vulnerability to a MDE during and after the menopausal transition was not accounted for by frequent VMS or by levels of or changes in reproductive hormones. Lack of a statistically significant association between frequent VMS and MDE may be due to the short time frame over which we assessed VMS (i.e., past 2 weeks). It is possible that a measure of VMS frequency over a longer period of time may have been more strongly related to major depression onset in the multivariate analysis and may have attenuated the association between menopausal status and MDE, particularly because VMS may wax and wane across a period of time. Nonetheless, future studies should continue to examine the role of VMS in the development of MDEs.

We found no significant associations between any reproductive hormone and MDEs which is consistent with the literature showing no direct associations between depression and serum estradiol (E2) levels (Schmidt et al, 2002). However, single annual hormone samples have limited ability to provide information about the underlying hormonal dynamics that occur during the menopausal transition. Reproductive hormone secretion, especially that of estradiol, is subject to great variability during the menopausal transition. It has been hypothesized that the fluctuations in reproductive hormones in susceptible women pose a risk for depression. However, we found no association between change or variability in hormones over time with major depression. In contrast, the POA study of first onset depression did report an association between the variability of E2 and FSH measured at two consecutive monthly assessments with high depressive symptoms or depressive disorder (Freeman et al, 2006). Our null result for testosterone was in contrast to our previously reported associations between high depressive symptom scores (CES-D $\geq 16$ ) and testosterone levels and changes in these over the first eight years of SWAN in the entire cohort. The conflicting findings may be due to the relatively small sample of women in the current analysis $(n=221)$ compared to the SWAN cohort of more than 3,000 women. Perhaps, $\mathrm{T}$ is more likely to be associated with the level of depressive symptoms than to the syndromal cluster represented by MDE.

It is noteworthy that the odds of a MDE were nearly tripled in those with a history of major depression at baseline or at least one very upsetting event at the annual visits (ORs=2.83 and 2.93, respectively) indicating that in addition to and independent of menopausal status, lifetime depression history and the experience of recent life stressors made significant contributions to the risk of a MDE. Both factors are known to be consistently related to major depression (Kendler \& Gardner, 2010) but prior studies of menopause have not assessed their importance relative to the menopausal transition.

In addition to the limitations concerning the measures of VMS and hormones described above, other limitations of the current report are important. Because of the small size of the sample, we were limited in our ability to examine racial/ethnic differences in major depression according to menopausal status and/or history of major depression at study entry. However, in a future paper encompassing a longer period of follow-up we will focus on the characteristics and subgroups of midlife women that may be particularly vulnerable to a 
MDE. Second, structured interviews, although they improve diagnostic reliability and validity, are subject to subjective recall and interpretation of symptoms.

Finally, we do not know the potential bias introduced by the absence of data on women whom we could not contact or who refused to complete the initial telephone screener. If this group of women included more who were depressed at the time of contact than did the group who participated, there would likely be more cases of major depression during the study. Because our sample is not large enough to determine whether women with a prior history of depression would be more likely to experience a subsequent MDE when they were peri- or post menopausal than premenopausal, we cannot know how our current findings would be affected.

The current study is unique in its ability to assess major depression annually in midlife African American and Caucasian women as they transitioned through perimenopause and postmenopause. We used a standard semi-structured interview to assess MDE during the time between visits and currently which allowed us to account for episodes for the entire 10year period. The current findings confirm the greater risk for MDEs during the menopausal transition than before. While our data also indicate that the years immediately after the transition are a high risk period for MDEs, these findings warrant confirmation when all SWAN participants become late postmenopausal. Many questions remain regarding the factors that contribute to the elevated risk of MDEs during the menopausal transition and after including the role of reproductive hormone changes and the characteristics of the subgroup of vulnerable women. When all women enrolled in our study complete the menopausal transition, we will be able to address these important questions in further detail.

\section{Acknowledgments}

The Study of Women's Health Across the Nation (SWAN) has grant support from the National Institutes of Health (NIH), DHHS, through the National Institute on Aging (NIA), the National Institute of Nursing Research (NINR), the National Institute of Mental Health (NIMH) and the NIH Office of Research on Women's Health (ORWH) (Grants NR004061; AG012505, AG012535, AG012531, AG012539, AG012546, AG012553, AG012554, AG012495, MH59689). The content of this article is solely the responsibility of the authors and does not necessarily represent the official views of the NIA, NINR, ORWH or the NIH.

Clinical Centers: University of Michigan, Ann Arbor-MaryFran Sowers, PI; Massachusetts General Hospital, Boston, MA - Joel Finkelstein, PI 1999 - present; Robert Neer, PI 1994 - 1999; Rush University, Rush University Medical Center, Chicago, IL - Howard Kravitz, PI 2009 - present; Lynda Powell, PI 1994 - 2009; University of California, Davis/Kaiser - Ellen Gold, PI; University of California, Los Angeles - Gail Greendale, PI; Albert Einstein College of Medicine, Bronx, NY - Rachel Wildman, PI 2010; Nanette Santoro, PI 2004 - 2010; University of Medicine and Dentistry - New Jersey Medical School, Newark - Gerson Weiss, PI 1994 - 2004; and the University of Pittsburgh, Pittsburgh, PA - Karen Matthews, PI.

NIH Program Office: National Institute on Aging, Bethesda, MD - Sherry Sherman 1994 - present; Marcia Ory 1994 - 2001; National Institute of Nursing Research, Bethesda, MD - Project Officer.

Central Laboratory: University of Michigan, Ann Arbor - Daniel McConnell (Central Ligand Assay Satellite Services).

Coordinating Center: University of Pittsburgh, Pittsburgh, PA - Kim Sutton-Tyrrell, PI 2001 - present; New England Research Institutes, Watertown, MA - Sonja McKinlay, PI 1995 - 2001.

Steering Committee: Susan Johnson, Current Chair

Chris Gallagher, Former Chair

We thank the study staff at each site and all the women who participated in SWAN. 


\section{References}

Avis NE, Brambilla D, McKinlay SM, Vass K. A longitudinal analysis of the association between menopause and depression. Results from the Massachusetts Women's Health Study. Annals of Epidemiology. 1994; 4:214-220. [PubMed: 8055122]

Bromberger JT, Matthews KA, Schott LL, Brockwell S, Avis N, Kravitz HM, Everson-Rose SA, Gold EB, Sowers M, Randolph J Jr. Depressive symptoms during the menopausal transition: the Study of Women's Health Across the Nation (SWAN). Journal of Affective Disorder. 2007; 103:267-272.

Burger HG, Dudley EC, Hopper JL, Groome N, Guthrie JR, Green A, Dennerstein L. Prospectively easured levels of Serum Follicle-stimulating hormone, estradiol, and the dimeric inhibins during the menopausal transition in a population-based cohrt of women*. Journal of Clinical Endocrinology \& Metabolism. 1999; 84:4025-4030. [PubMed: 10566644]

Cohen LS, Soares CN, Vitonis AF, otto MW, Harlow BL. Risk for new onset of depression during the menopausal transition: The Harvard study of moods and cycles. Archives of General Psychiatry. 2006; 63:385-390. [PubMed: 16585467]

Freeman EW, Sammel MD, Lui L, Gracia CR, Nelson DB, Hollander L. Hormones and menopausal status as predictors of depression in women in transition to menopause. Archives of General Psychiatry. 2004; 61:62-70. [PubMed: 14706945]

Freeman EW, Sammel MD, Lin H, Nelson DB. Associations of hormones and menopausal status with depressed mood in women with no hisotry of depression. Archives of General Psychiatry. 2006; 63:375-382. [PubMed: 16585466]

Hunter M. The southeast England longitudinal study of the climacteric and postmenopause. Maturitas. 1992; 14:117-126. [PubMed: 1565020]

Kaufert PA, Gilbert P, Tate R. The Manitoba Project: a re-examination of the link between menopause and depression. Maturitas. 1992; 114:143-155. [PubMed: 1565022]

Kendler KS, Gardner CO. Dependent stressful life events and prior depressive episodes in the prediction of major depression. Archives of General Psychiatry. 2010; 67:1120-1127. [PubMed: 21041613]

Kuh DL, Wadsworth M, Hardy R. Women's health in midlife: the influence of the menopause, social factors and health in earlier life. British Journal of Obstetrics and Gynecology. 1997; 104:923933.

Maarten LW, Knottnerus JA, Pop VJ. Menopausal transition and increased depressive symptomatology: a community based prospective study. Maturitas. 2002; 42:195-200. [PubMed: 12161043]

Matthews, KA.; Bromberger, JT.; Egland, G. behavioral antecedents and consequences of the menopause. In: Korenman, SG., editor. The Menopause. Norwell, MA: Serono Symposia; 1990. p. $1-15$.

Matthews KA, Wing RR, Kuller LH, Leilahn EN, Plantinga P. Influence of the perimenopause on cardiovascular risk factors and symptoms of middle-aged healthy women. Archives of Internal Medicine. 1994; 20:2349-2355. [PubMed: 7944857]

McKinlay SM, Jefferys M. The menopausal syndrome. British Journal of Preventive and Social Medicine. 1974; 28:108-115. [PubMed: 4854952]

Neugarten BL, Kraines RJ. "Menopausal Symptoms" in Women of Various Ages. Psychosomatic Medicine. 1965; 27:266-273. [PubMed: 14327878]

O'Connor VM, Del Mar CB, Sheehan M, Siskind V, Fox-Young S, Cragg C. Do psychosocial factors contribute more to symptom reporting by middle-aged women than hormonal status? Maturitas. 1994; 20:63-69. [PubMed: 7715476]

Randolph JF, Sowers MF, Bondarenko I, Gold EB, Greendale GA, Bromberger JT, Brockwell SE, Matthews KA. The relationship change in reproductive hormones and vasomotor symptoms during the menopausal transition. Journal of Clinical Endocrinology and Metabolism. 2005; 90:61066112. [PubMed: 16144949]

Schmidt PJ, Murphy JH, Haq N, Danaceau MA, St. Clair LS. Basal plasma hormones levels in depressed perimenopausal women. Psychoneuroendocrinology. 2002; 27:907-920. [PubMed: 12383452] 
Schmidt PJ, Nazli H, Rubinow DR. A longitudinal evaluation of the relationship between reproductive status and mood in perimenopausal women. America Journal of Psychiatry. 2004; 161:2238-2244.

Sowers, MF.; Crawford, S.; Sternfeld, B.; Morganstein, D.; Gold, EB.; Greendale, GA.; Evans, D.; Neer, R.; Matthews, KA.; Sherman, S.; Lo, A.; Weiss, G.; Kelsey, J. SWAN: a multi-center, multiethnic, community-based cohort study of women and the menopause. In: Lobo, R.; Kelsey, J.; Marcus, R., editors. Menopause: Biology and Pathobiology. San Diego, CA: Academic Press; 2000. p. 175-178.

Spitzer RL, William JB, Gibbon M, First MB. The Structured Clinical Interview for DSM-III-R (SCID). I: History, rationale, and description. Archives of General Psychiatry. 1992; 49:624-629. [PubMed: 1637252]

Weissman M. The Myth of Involutional Melancholia. Journal of the American Medical Association. 1979; 242:742-744. [PubMed: 459064]

Williams JB, Gibbon M, First MB, Spitzer RL, Davies M, Borus J, howes MJ, Kane J, Pope HG Jr, Rounsaville B. The Structured Clinical Interview for DSM-III-R (SCID). II: Multisite test-retest reliability. Archives of General Psychiatry. 1992; 49:630-636. [PubMed: 1637253]

World Health Organization Scientific Group. Research on the Menopause in the 1990's. Geneva, Switzerland: World Health Organization; 1996. p. 866WHO Technical Report Series 


\section{Number of Observations Z Zumber of Major Depressive Episodes}

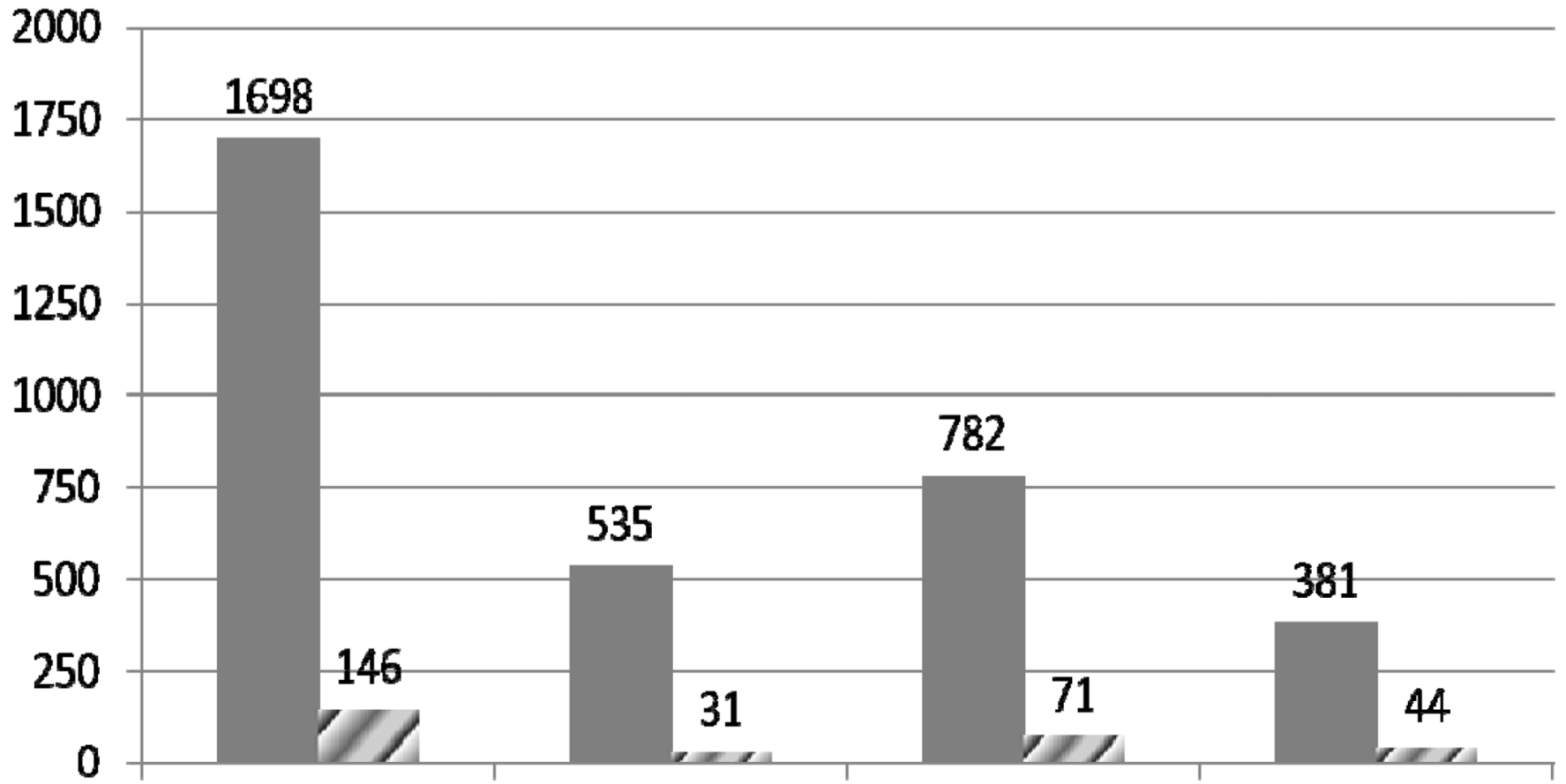

Total Premenopause Perimenopause Postmenopause

Figure 1.

Number of Person-Years of Observations (visits) for Total Sample (maximum possible $=1913$ ), and for Premenopause, Perimenopause, and Postmenopause Visits. 
है

界

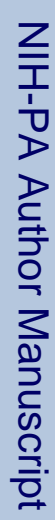

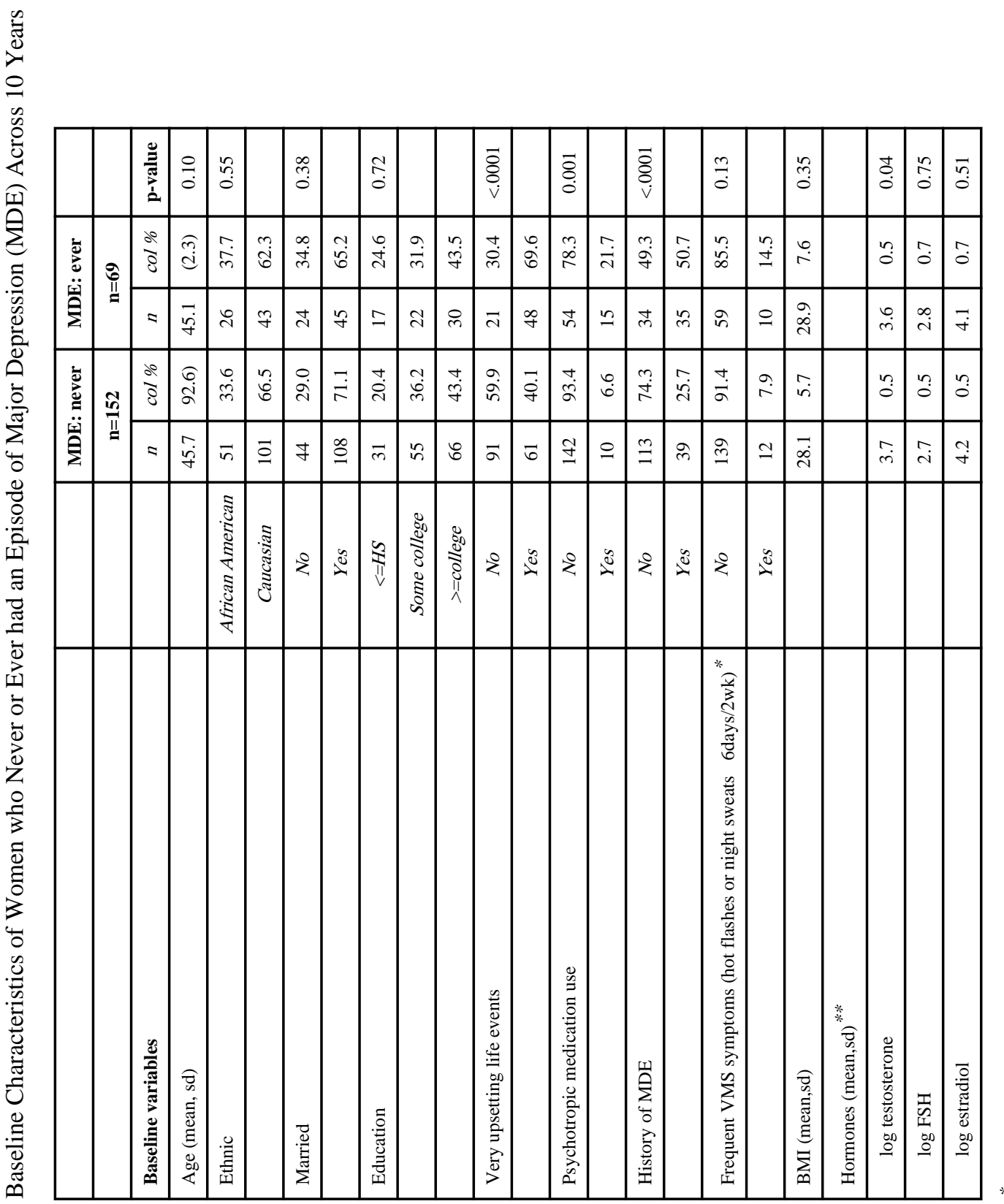

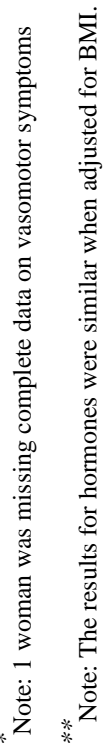


Table 2

Univariate Repeated Measures Associations of Baseline and Annual Measures with Odds of a Major Depressive Episode from Baseline Through Visit 9

\begin{tabular}{|c|c|c|c|}
\hline & OR & $95 \% \mathrm{CI}$ & p-value \\
\hline \multicolumn{4}{|l|}{ Baseline characteristics } \\
\hline Age & 1.10 & $(1.03,1.17)$ & 0.005 \\
\hline African American & 1.62 & $(0.76,3.46)$ & 0.21 \\
\hline History of major depressive disorder & 4.51 & $(2.20,9.25)$ & $<.0001$ \\
\hline \multicolumn{4}{|l|}{ Annual measures } \\
\hline \multicolumn{4}{|l|}{ Menopausal status (referent category: premenopause) } \\
\hline Perimenopause & 2.17 & $(1.23,3.81)$ & 0.007 \\
\hline Postmenopause & 3.43 & $(1.79,6.56)$ & $<.0001$ \\
\hline Hormone therapy use & 1.20 & $(0.38,3.73)$ & 0.76 \\
\hline 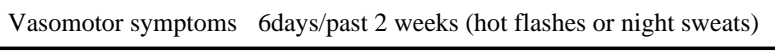 & 2.50 & $(1.48,4.22)$ & 0.001 \\
\hline BMI & 1.08 & $(1.02,1.13)$ & 0.004 \\
\hline $\log \mathrm{E} 2 * *$ & 0.82 & $(0.63,1.06)$ & 0.13 \\
\hline $\log \mathrm{T}^{* *}$ & 1.04 & $(0.62,1.74)$ & 0.90 \\
\hline $\log \mathrm{FSH}^{* *}$ & 1.25 & $(.97,1.62)$ & 0.08 \\
\hline Change in E2 from baseline ${ }^{*}$ & 1.00 & $(1.00,1.00)$ & 0.57 \\
\hline Change in Testosterone from baseline ${ }^{*}$ & 1.01 & $(1.00,1.03)$ & 0.10 \\
\hline Change in FSH from baseline * & 1.00 & $(0.99,1.01)$ & 0.90 \\
\hline
\end{tabular}

* Adjusted for baseline value

*** Note: The results for hormones were similar when adjusted for BMI. 
Table 3

Results of Random Effects Logistic Regression Analyses for Odds of Major Depressive Episode (MDE) from Baseline Through Visit 9 by Menopausal Status

\begin{tabular}{|l|l|l|l|}
\hline & $\begin{array}{l}\text { Model A } \\
\text { OR } \mathbf{9 5 \%} \text { CI) }\end{array}$ & $\begin{array}{l}\text { Model B } \\
\text { OR }(\mathbf{9 5 \%} \text { CI) }\end{array}$ & $\begin{array}{l}\text { Model C } \\
\text { OR (95\% CI) }\end{array}$ \\
\hline Variables & & & \\
\hline Age & $0.99(0.90,1.09)$ & $0.96(0.86,1.06)$ & $0.95(0.86,1.05)$ \\
\hline Menopausal status (ref=pre) & & & \\
\hline \multicolumn{1}{|c|}{ Perimenopause } & $2.20(1.17,4.16)$ & $2.08(1.06,4.08)$ & $1.98(1.00,3.92)$ \\
\hline \multicolumn{1}{|c|}{ Postmenopause } & $3.57(1.35,9.39)$ & $4.32(1.54,12.13)$ & $3.86(1.36,10.92)$ \\
\hline African American & $1.96(0.96,3.97)$ & $1.64(0.82,3.29)$ & $1.56(0.78,3.15)$ \\
\hline History of major depression at baseline & & $3.02(1.57,5.80)$ & $2.98(1.55,5.72)$ \\
\hline Psychotropic medication use & & $4.71(2.65,8.38)$ & $4.55(2.55,8.09)$ \\
\hline Very upsetting life event & & $2.66(1.59,4.47)$ & $2.62(1.56,4.40)$ \\
\hline BMI continuous & & $1.04(.099,1.09)$ & $1.04(1.00,1.09)$ \\
\hline Frequent VMS $(ð 6$ days/past 2 weeks) & & & $1.76(0.99,3.11)$. \\
\hline
\end{tabular}

Model A: adjusted for age and race

Model B: addition of history of major depression at baseline, annual psychotropic medication, annual very upsetting life events, BMI Model C: final model with addition of annual frequent VMS 